\title{
Medulloblastoma with Extensive Nodularity
}

National Cancer Institute

\section{Source}

National Cancer Institute. Medulloblastoma with Extensive Nodularity. NCI Thesaurus.

Code C5407.

A medulloblastoma characterized by nodularity and neuronal differentiation. 